\title{
O carvedilol no tecido conjuntivo cardíaco de ratos durante o desenvolvimento da hipertrofia miocárdica concêntrica.
}

\author{
Carvedilol in conjuctive tissue of rats during hypertrophic cardiomyopathy
}

\author{
Moacir Leomil Neto ${ }^{1}$ Lívia Aparecida D’Avila Bitencourt Pascoal ${ }^{2}$ \\ Júlio César Carvalho Balieiro ${ }^{3}$ Maria Helena Matiko Akao Larsson ${ }^{3}$ \\ Pedro Primo Bombonato ${ }^{3}$ Hildebrando Gomes Benedicto ${ }^{4}$ \\ Lílian Barreto Elton ${ }^{5}$
}

\section{RESUMO}

A estimulação catecolaminérgica precoce e excessiva do coração, que ocorre na insuficiência cardíaca congestiva, acentua a sobrecarga de pressão e o volume nos ventrículos, agravando, conseqüentemente, a isquemia miocárdica, causando morte de miócitos com deposição de tecido conjuntivo e progressiva redução do débito cardíaco. $O$ carvedilol, um $\beta$ e $\alpha 1$-bloqueador não cardio-seletivo, vem sendo associado a melhorias na função miocárdica e à regressão da hipertrofia ventricular esquerda. O objetivo do presente trabalho foi avaliar a ação do carvedilol em ratos submetidos a um modelo experimental de hipertrofia miocárdica por sobrecarga de pressão, por meio da coartação da aorta subdiafragmática. Ratos Wistar (65 animais) foram divididos em quatro grupos: não coartados/tratados com placebo, coartados/tratados com placebo, coartados/tratados com $0,1 \mathrm{mg} \mathrm{kg}^{-1}$ de carvedilol e coartados/tratados com $1 \mathrm{mg} \mathrm{kg}^{-1}$ de carvedilol. Após o procedimento cirúrgico e 20 dias de tratamento, os animais foram submetidos à eutanásia $e$ coletados fragmentos que, depois de fixados em formol a $10 \%$ e processados pela técnica histológica convencional utilizando coloração picrossirius, permitiram contagem de tecido conjuntivo nos ventrículos esquerdo e direito. Resultados: nos animais coartados/tratados com placebo, houve aumento na porcentagem de tecido conjuntivo nos ventrículos esquerdo $e$ direito; nos coartados/tratados com $0,1 \mathrm{mg} \mathrm{kg}^{-1}$ de carvedilol, houve uma redução parcial da quantidade de tecido colágeno do ventrículo esquerdo e direito, e, nos coartados/tratados com $1 \mathrm{mg} \mathrm{kg}^{-1}$ de carvedilol, houve uma redução significativa na quantidade de tecido colágeno do ventrículo esquerdo.
Palavras-chave: carvedilol, hipertrofia miocárdica, ratos, coartação.

\begin{abstract}
Early and excessive catecholamine stimulation of the heart increases the overcharge blood pressure and volume on ventricles. Thus worsening myocardial ischemia by causing the death of myocytes. With connective tissue deposition and progressive reduction of heart output. Carvedilol, a $\beta$ and $\alpha 1$ blocker non heart-selective, has been associated to improvement on myocardial function on left ventricle hypertrophy regression. The aim of this study was to evaluate the action of carvedilol in rats undergone to an experimental model of hypertrophic cardiomyopathy by pressure overcharge, through subdiaphragmatic aortic coarctation. Wistar rats (65 animals) divided into four groups: non- coarcted/ treated with placebo, coarcted / treated with placebo, coarcted / treated with $0.1 \mathrm{mg}$ $\mathrm{kg}^{-1}$ of carvedilol and coarcted / treated with $1 \mathrm{mg} / \mathrm{kg}$ of carvedilol. After surgery and 20 days of treatment, the animals were euthanized and samples were collected, fixed in formol $10 \%$ and processed using picrossirius staining to quantify connective tissue on left and right ventricles. On those coarcted animals / treated with placebo, there was a rise on the percentage of connective tissue on left and right ventricles; on coarcted animals / treated with $0.1 \mathrm{mg} \mathrm{kg}^{-1}$ of carvedilol there was a partial reduction on the amount of collagen tissue of left and right ventricles; and on those coarcted animals / treated with $1 \mathrm{mg} \mathrm{kg}^{-1}$ of carvedilol there was a significant reduction on the amount of collagen tissue of left ventricle.
\end{abstract}

${ }^{1}$ Curso de Medicina Veterinária, Pontifícia Universidade Católica de Minas Gerais (PUC) e Centro Universitário da Fundação Otávio Bastos (UNIFEOB). Rua Caminho Cinco, n. 20, Bairro Bortolan, 37704-403, Poços de Caldas, MG, Brasil. E-mail: mleomil@pucpcaldas.br. Autor para correspndência.

${ }^{2}$ Curso de Medicina Veterinária, UNIFEOB. Av. Otávio Bastos, São João da Boa Vista, SP, Brasil.

${ }^{3}$ Universidade de São Paulo (USP). Av. Prof. Dr Orlando Marques de Paiva, 87, São Paulo, SP, Brasil.

${ }^{4}$ Programa de Pós-graduação, USP. Av. Prof. Dr Orlando Marques de Paiva 87, São Paulo-SP-Brasil.

${ }^{5}$ Curso de Medicina Veterinária, PUC de Minas Gerais. Av. Padre Francis Cletus Cox, 1661, Poços de Caldas, MG, Brasil. 
Key words: carvedilol, myocardial hypertrophy, rats, coarctation.

\section{INTRODUÇÃO}

Relatos da literatura indicam que, na hipertensão arterial sistêmica, na estenose aórtica valvar e na coartação da aorta, a hipertrofia ventricular esquerda caracteriza-se por crescimento das miocélulas, acompanhado de acúmulo, proporcional ou não, de colágeno (CHEITLIN et al., 1980; GASPARI et al., 1985). Um estudo realizado com o coração de 127 pacientes, provenientes de autópsia do Hospital Johns Hopkins, em Baltimore - USA, demonstrou que a hipertrofia das células musculares cardíacas é geralmente acompanhada por aumento do tecido conjuntivo (MOORE et al., 1980).

O estudo histológico e ultraestrutural dos corações de gatos com cardiomiopatia hipertrófica revelou a presença de uma marcada fibrose intersticial no miocárdio ventricular esquerdo, no qual se observou uma extensa deposição das fibras colágenas associadas a fibroblastos (VAN-VLEET et al., 1980).

Autores afirmam que a deposição de tecido conjuntivo, a hipertrofia e o remodelamento cardíaco são respostas compensatórias do miocárdio ao trabalho aumentado, pois a hiperfunção miocárdica induz à injúria miocárdica e ao aumento de tamanho do miócito (hipertrofia celular por meio da adição de sarcômeros), o que provoca um aumento na massa e no tamanho generalizado do coração (COTRAN et al., 1996; ROSSI, 1998).

Em felinos, uma das principais causas da cardiomiopatia hipertrófica é a falha congênita na formação das estruturas contráteis de alguns miócitos que, conseqüentemente, sobrecarregam os miócitos normais, provocando sua hipertrofia (LASTE, 2001).

Experimentalmente, a coartação da aorta subdiafragmática é um modelo previamente descrito de cardiomiopatia hipertrófica (SANTOS et al., 1998) que se caracteriza por elevação da pressão arterial sistêmica dos ratos em aproximadamente $28 \%$ em relação à pressão normal, elevando, dessa forma, a póscarga e o trabalho cardíaco, os quais resultam em injúria, hipertrofia e remodelamento miocárdico (SANTOS et al., 1998). Quanto maior o trabalho cardíaco e, conseqüentemente, os danos causados ao miocárdio, maior será a deposição de tecido conjuntivo (colágeno) entre as fibras musculares, sendo, dessa forma, a porcentagem de tecido colágeno no miocárdio representativa do grau de injúria ao qual ele foi submetido (ABRAHAMS et al., 1987).
Em vários processos patológicos, principalmente cardiopatias, o Sistema Nervoso Autônomo Simpático (SNAS) atua como um dos mecanismos compensatórios fisiológicos para a manutenção do débito cardíaco. Porém, a partir de certo grau de insuficiência cardíaca, os efeitos hipertensivos cronotrópico e inotrópico positivo do SNAS geram graves alterações cardíacas como a sobrecarga de pressão e volume nos ventrículos, conseqüente isquemia miocárdica, morte de miócitos com deposição de tecido conjuntivo, diminuição da contratilidade cardíaca e nova sobrecarga de pressão e volume, contribuindo, dessa forma, para a progressiva redução do débito cardíaco da hipertrofia e do remodelamento das câmaras cardíacas (LEFKOWITZ et al., 1996; KITTLESON \& KIENLE, 1998).

O carvedilol é um $\beta$-bloqueador não seletivo que bloqueia competitivamente os receptores $\beta 1, \beta 2$ e $\alpha 1$ (McTAVISH, et al., 1993, HATTORI et al., 1989, LASTE, 2001; VITAL, 2002). Carece de atividade simpatomimética intrínseca e possui propriedades inotrópicas e cronotrópicas negativas e vasodilatadoras, principalmente através do bloqueio $\alpha 1$ (MOORE et al., 1980). O carvedilol reduz também a área de fibrose miocárdica (WATANABE et al., 2003), apresenta atividade antioxidante (LASTE, 2001), remove diretamente radicais livres de oxigênio, previne peroxidação lipídica nas membranas cardíacas, previne a depleção dos antioxidantes e protege células em cultura da lesão induzida por radicais livres, atenuando os efeitos deletérios da doxorrubicina sobre o miocárdio (SAWANGKOON et al., 2000).

Estudos demonstram que, em pacientes que recebem tratamento convencional para insuficiência cardíaca congestiva, tendo sido acompanhados por um período médio de seis meses, o carvedilol diminuiu a taxa de mortalidade em mais de 60\% (HOFFMAN \& LEFKOWITZ, 1996). Trabalhos também demonstram que o carvedilol protege o coração contra necrose, arritmia e reduções na freqüência da arritmia induzida pela isquemia ou digitálicos (BRAUNWALD et al., 1996).

Efeitos benéficos do carvedilol nos sintomas, classe funcional, função e remodelamento ventricular em humanos com insuficiência cardíaca refratária foram relatados por BOCCHI et al. (1998). Em 1996, um ensaio duplo-cego, controlado com placebo e envolvendo 1.094 homens e mulheres com insuficiência cardíaca classes II, III e IV, e fração de ejeção menor ou igual a 0,35, demonstrou, consistentemente, os efeitos benéficos do carvedilol na redução da morbidade e da mortalidade. 
O carvedilol tem sido associado à melhora na função miocárdica, promovendo melhora na póscarga com redução do volume ventricular esquerdo (KATZUNG, 2006). Foi observada regressão da hipertrofia ventricular esquerda em pacientes com hipertensão essencial de leve à moderada (WHY \& RICHARDSON, 1992).

O presente trabalho teve por escopo avaliar a ação do bloqueio dos receptores $\beta 1, \beta 2$ e $\alpha 1$ adrenérgico (carvedilol) em ratos com hipertrofia miocárdica concêntrica, por sobrecarga de pressão, causada pela coartação da aorta subdiafragmática por meio da histomorfometria de tecido conjuntivo na parede livre dos ventrículos esquerdo e direito.

\section{MATERIAL E MÉTODO}

Foram utilizados 65 ratos Wistar, machos e fêmeas, pesando entre 350 e 450 gramas, mantidos à temperatura controlada $\left(20\right.$ a $\left.23^{\circ} \mathrm{C}\right)$ por meio de aparelhos de ar condicionado, com ciclos de luz de $12 \mathrm{~h}$ de claro/escuro. Água e comida foram fornecidas ad libitum. Todos os procedimentos experimentais observaram as normas éticas relativas ao uso de animais de experimentação.

Os animais foram alocados em quatro grupos comparativos distintos: G1 - 19 animais sem a coartação da aorta subdiafragmática e tratamento com placebo (solução fisiológica); G2 - 12 animais submetidos à coartação da aorta subdiafragmática e tratamento com placebo (solução fisiológica); G3 - 23 animais submetidos à coartação da aorta subdiafragmática e tratados com $0,1 \mathrm{mg} \mathrm{kg}^{-1}$, via oral, de carvedilol e G4 - 11 animais submetidos à coartação da aorta subdiafragmática e tratados com $1 \mathrm{mg} \mathrm{kg}^{-1}$, via oral, de carvedilol.

Os ratos dos grupos G2, G3 e G4 foram inicialmente anestesiados (associação anestésicaXilazina (20 $\mathrm{mg} \mathrm{ml}^{-1}$ ), Quetamina (50mg ml-1) e Bupremorfina (30 $\mathrm{mg} \mathrm{ml}^{-1}$ )) e mantidos em respiração espontânea com ar ambiente. Após iniciar o efeito anestésico, foi realizada a coartação da aorta subdiafragmática, conforme descrito por SANTOS et al. (1998). Os animais foram tratados com antibiótico (0,03ml, penicilina benzatina, SC) e tiveram acesso livre à água e comida. Os animais do grupo G1 foram submetidos à mesma associação anestésica e à realização da incisão e da sutura, porém não se realizou a coartação.

Após a reabilitação, os animais dos grupos G1 e G2 foram submetidos a tratamentos diários, durante 20 dias, com solução fisiológica (placebo), por via oral, a cada 24 horas, e os dos grupos G3 e G4 foram submetidos a tratamento com carvedilol a cada 24 horas.

Após os vinte dias de tratamento com placebo ou carvedilol, os animais dos quatro grupos (G1, G2, G3 e G4) foram pesados e submetidos à eutanásia com éter sulfúrico. Realizou-se toracotomia, com posterior retirada do coração, onde os vasos da base foram seccionados próximos à sua origem. Para o estudo histológico, os corações coletados foram lavados e fixados em solução aquosa de formol a $10 \%$ para preparação, segundo técnica histológica convencional.

Nos corações, foram realizadas incisões transversais entre o terço proximal e o médio, e outra incisão entre o terço médio e o distal dos ventrículos, resultando, dessa forma, um fragmento de aproximadamente $0,5 \mathrm{~cm}$ de espessura, composto pela parede livre dos ventrículos esquerdo e direito e pelo septo interventricular. No corte histológico, a musculatura ventricular foi dividida em parede livre do ventrículo esquerdo e parede livre do ventrículo direito. Em cada corte histológico do miocárdio, 20 contornos de fibras musculares foram copiados e o diâmetro celular foi calculado pela média aritmética dos valores obtidos. Em seguida, o fragmento do miocárdio foi processado, segundo a seqüência histológica convencional: desidratação, diafanização e inclusão em blocos de Paraplast ${ }^{\circledR}$, obtendo-se blocos contendo o terço médio dos ventrículos. Foram selecionados três cortes com $5 \mu$ de espessura de cada bloco para confecção das lâminas, perfazendo um total de 183 lâminas. As lâminas foram coradas de acordo com a técnica de picrossirius, para evidenciação das fibras colágenas do tecido conjuntivo miocárdico.

As lâminas foram observadas com auxílio do microscópio óptico Axioscópio Zeiss ${ }^{\circledR}$ e os resultados analisados em um microcomputador com programa de morfometria específico KS-400 Zeiss ${ }^{\circledR}$, segundo o seguinte processo: 1 . captação da imagem, 2. calibração do equipamento, 3. engenharia da imagem, 4. identificação da freqüência da cor do tecido estudado por campo, 5. binarização da imagem, 6. mensuração dos campos e 7. listagem dos dados.

Padronizaram-se a leitura e a mensuração de 10 campos por lâmina, num total de 1200 campos mensurados de 120 lâminas. De cada grupo de cinco mensurações, foram retiradas médias que foram agrupadas, segundo a seqüência dos fragmentos. Para efeito de mensuração, foram desprezadas as áreas de tecido conjuntivo referentes ao epicárdio, no endocárdio e às margens das artérias ou veias. Posteriormente, os resultados obtidos foram agrupados segundo grupos experimentais, para comparação dos parâmetros entre as variáveis. 
Para obtenção das estatísticas descritivas, utilizou-se o procedimento PROC UNIVARIATE do programa Statistical Analysis System, versão 8.02 (SAS, 1995). O delineamento experimental utilizado foi o inteiramente casualizado, sendo que cada grupo comparativo (GC) representou um tratamento. Para essas análises estatísticas, realizou-se o procedimento PROC GLM do programa supracitado.

Ao verificar efeito significativo $(\mathrm{P}<0,05)$ na análise de variância entre os grupos comparativos para as diferentes variáveis estudadas, utilizou-se o Teste de Tukey para discriminar as diferenças e/ou igualdades entre as médias dos diferentes grupos avaliados.

Para investigar a relação entre as medidas macromorfométricas dos animais (peso do coração, peso do animal e relação peso do coração: peso do animal) e as contagens de tecido conjuntivo do ventrículo esquerdo e do direito (mínimo, média máxima), empregou-se a análise de correlação momento produto de Pearson, por meio do procedimento PROC CORR do programa anteriormente citado.

\section{RESULTADOS}

Ventrículo esquerdo (Tabela 1): após 20 dias da coartação da aorta subdiafragmática, observou-se um significativo aumento na porcentagem de fibras colágenas comparado à quantidade de tecido conjuntivo dos animais do G1 (normais), tanto na contagem mínima, de 0,3172 para 0,6525 , como na contagem máxima, de 0,8323 para 1,4208 , e na contagem média, de 0,5573 para 0,8823 (Tabela 1 ), demonstrando que o modelo experimental adotado foi adequado para produzir injúria e posterior deposição de colágeno. Quando os animais foram submetidos a 20 dias de coartação da aorta subdiafragmática, associada ao tratamento com carvedilol na dose de $0,1 \mathrm{mg} \mathrm{kg}^{-1}$, as porcentagens mínima, máxima e média de tecido colágeno encontradas na parede livre foram de 0,4715 ;
1,1510 e 0,7205, respectivamente, sendo esses valores estatisticamente iguais tanto ao grupo G1 (normais) como ao G2 (coartado). Já nos animais que foram submetidos a 20 dias de coartação da aorta subdiafragmática associada ao tratamento com carvedilol na dose de $1,0 \mathrm{mg} \mathrm{kg}^{-1}$ (G4), não se observou diferença na porcentagem de tecido colágeno em relação ao G1 (normal) e ao G3 (tratado $0,1 \mathrm{mg} \mathrm{kg}^{-1}$ ). Entretanto, houve uma diminuição significativa em relação aos animais coartados sem tratamento (G2), sendo a porcentagem mínima encontrada de 0,2980, a máxima de 0,8034 e a média de 0,4970.

Ventrículo direito (Tabela 2): após 20 dias da coartação da aorta subdiafragmática, observou-se um significativo aumento na porcentagem de fibras colágenas comparado à quantidade de tecido conjuntivo dos animais do G1 (normais), tanto na contagem mínima, que aumentou de 0,1994 para 0,3633, como na contagem máxima, de 0,4256 para 0,8683 , e na contagem média, de 0,3186 para 0,6262 (Tabela 2). Durante os 20 dias de coartação da aorta subdiafragmática, associada ao tratamento com carvedilol na dose de $0,1 \mathrm{mg} \mathrm{kg}^{-1}$, as porcentagens mínima, máxima e média de tecido colágeno encontradas na parede livre foram de 0,2913, 0,7045 e 0,4981, respectivamente, sendo as porcentagens mínima e média estatisticamente iguais às do grupo G1 (normais) e G2 (coartado) e a porcentagem máxima de tecido colágeno diferente ao grupo G1 (normais) e igual ao G2 (coartado). Nos animais que foram submetidos a 20 dias de coartação da aorta subdiafragmática, associada ao tratamento com carvedilol na dose de $1,0 \mathrm{mg} \mathrm{kg}^{-1}$ (G4), não se observou diferença na porcentagem de tecido colágeno em relação ao G1 (normal), G2 (coartado) e G3 (tratado $0,1 \mathrm{mg} \mathrm{kg}^{-1}$ ) na porcentagem mínima de tecido colágeno; na porcentagem máxima, houve igualdade em relação ao G1 (normal) e diferença estatística em relação ao G2 (coarctado) e G3 (tratado $0,1 \mathrm{mg} \mathrm{kg}^{-1}$ ); em relação à porcentagem média, houve

Tabela 1 - As porcentagens mínima, média e máxima de tecido colágeno na parede livre do ventrículo esquerdo, segundo os grupos comparativos avaliados.

\begin{tabular}{|c|c|c|c|c|}
\hline & G2-coartado(N=12) & G3-tratado $0,1 \mathrm{mg} \mathrm{kg}^{-1}(\mathrm{~N}=23)$ & G4-tratado $1,0 \mathrm{mg} \mathrm{kg}^{-1}(\mathrm{~N}=11)$ & G1-normal $(\mathrm{N}=19)$ \\
\hline Min. VE & $0,6525^{a}$ & $0,4715^{\mathrm{a}, \mathrm{b}}$ & $0,2980^{\mathrm{b}}$ & $0,3172^{b}$ \\
\hline Med. VE & $0,8823^{\mathrm{a}}$ & $0,7205^{\text {a, b }}$ & $0,4970^{b}$ & $0,5573^{b}$ \\
\hline Máx. VE & $1,4208^{\mathrm{a}}$ & $1,1510^{\mathrm{a}, \mathrm{b}}$ & $0,8034^{\mathrm{b}}$ & $0,8323^{b}$ \\
\hline
\end{tabular}

Médias seguidas por uma mesma letra, em uma mesma linha, não diferem entre si pelo teste de Turkey $(\mathrm{P}>0,05)$.

Min. VE - porcentagem mínima de tecido colágeno na parede livre do ventrículo esquerdo.

Máx. VE - porcentagem máxima de tecido colágeno na parede livre do ventrículo esquerdo.

Med. VE - porcentagem média de tecido colágeno na parede livre do ventrículo esquerdo. 
Tabela 2 - As porcentagens mínima, média e máxima de tecido colágeno na parede livre do ventrículo direito, segundo os grupos comparativos avaliados.

\begin{tabular}{|c|c|c|c|c|}
\hline & G2- coarctado $(\mathrm{N}=12)$ & G3-tratado $0,1 \mathrm{mg} \mathrm{kg}^{-1}(\mathrm{~N}=23)$ & G4-tratado $1,0 \mathrm{mg} \mathrm{kg}^{-1}(\mathrm{~N}=11)$ & G1-normal(N=19) \\
\hline Min. VD & $0,3633^{a}$ & $0,2913^{\mathrm{a}, \mathrm{b}}$ & $0,2315^{\mathrm{a}, \mathrm{b}}$ & $0,1994^{\mathrm{b}}$ \\
\hline Med. VD & $0,6262^{a}$ & $0,4981^{\mathrm{a}, \mathrm{b}}$ & $0,3011^{\mathrm{b}}$ & $0,3186^{b}$ \\
\hline Máx. VD & $0,8683^{a}$ & $0,7045^{\mathrm{a}}$ & $0,4075^{b}$ & $0,4256^{\mathrm{b}}$ \\
\hline
\end{tabular}

Médias seguidas por uma mesma letra, em uma mesma linha, não diferem entre si pelo teste de Turkey $(\mathrm{P}>0,05)$.

Min. VD - porcentagem mínima de tecido colágeno na parede livre do ventrículo direito.

Máx. VD - porcentagem máxima de tecido colágeno na parede livre do ventrículo direito.

Med. VD - porcentagem média de tecido colágeno na parede livre do ventrículo direito.

uma diminuição significativa apenas em relação aos animais coartados sem tratamento (G2), sendo que a porcentagem mínima encontrada foi 0,2315, a máxima de 0,4075 e a média de 0,3011.

As estimativas de correlação entre o peso do animal, peso do coração e relação peso do coração: peso do animal com as porcentagens de tecido conjuntivo no miocárdio, tanto do ventrículo direito como no esquerdo, variaram de - 0,00348 (entre relação peso do coração:peso do animal e porcentagem mínima de tecido colágeno na parede livre do ventrículo direito) a 0,3131 (peso do coração e porcentagem máxima de tecido colágeno na parede livre do ventrículo direito).

\section{DISCUSSÃO}

O significativo aumento da porcentagem de tecido conjuntivo, especificamente o colágeno, nas paredes dos ventrículos esquerdo e direito, tanto na contagem mínima, quanto na máxima e na média, observado nos animais submetidos à coartação da aorta subdiafragmática e sem tratamento (G2 - coarctado) em relação aos não coartados (G1 - normal), demonstra que o modelo experimental adotado conseguiu mimetizar a hipertrofia ventricular e o acúmulo de colágeno, proporcional ou não, relatado na literatura em casos de hipertensão arterial sistêmica, estenose aórtica valvar e de cardiomiopatia hipertrófica (CHEITLIN et al., 1980; GASPARI et al., 1985). Os dados demonstram que os 20 dias de coartação, definidos para a evolução das alterações miocárdicas, foram suficientes para permitir que a sobrecarga de pressão (aumento da pós-carga) provocasse injúria, perda de miócitos e conseqüente deposição de tecido conjuntivo no interstício miocárdico.

Quando os animais coartados foram submetidos ao tratamento com carvedilol, na dosagem de $0,1 \mathrm{mg} \mathrm{kg}^{-1}$ a cada 24 horas, durante 20 dias (G3), observou-se que todas as contagens de tecido colágeno do ventrículo esquerdo eram, ao mesmo tempo, significativamente iguais às contagens de tecido conjuntivo do G1 (normal) e do G2 (coartado), demonstrando que houve uma parcial redução na quantidade de tecido conjuntivo. Isso permite concluir que o tratamento com carvedilol $\left(0,1 \mathrm{mg} \mathrm{kg}^{-1}\right.$, a cada 24 horas durante 20 dias) reduziu, mesmo que parcialmente, a injúria miocárdica do ventrículo esquerdo.

No grupo G4 (1,0mg kg-1, a cada 24 horas), os animais foram tratados com uma dose dez vezes maior que a do grupo G3 (tratado com $0,1 \mathrm{mg} \mathrm{kg}^{-1}$ ) e nas três contagens realizadas (mínimo, médio e máximo) no ventrículo esquerdo, não houve diferença significativa $(\mathrm{P}>0,05)$ entre estes grupos e o grupo G1 (normal). Entretanto, foi verificada diferença significativa $(\mathrm{P}<0,05)$ em relação ao G2 (coartado), demonstrando que, nessa dose, houve uma significativa redução da porcentagem de tecido conjuntivo no miocárdio.

$\mathrm{O}$ retorno às porcentagens normais de tecido conjuntivo no miocárdio, após tratamento com carvedilol, demonstra que sua ação vasodilatadora, inotrópica/cronotrópica negativa, sua ação antioxidante ou mesmo o conjunto dessas ações (HATTORI et al., 1989; LASTE, 2001; WATANABE et al., 2003) aumentaram a viabilidade e a resistência dos miócitos frente às agressões impostas por doenças circulatórias e/ou cardíacas. Assim, reduziram a injúria miocárdica e a deposição de tecido colágeno no coração e, conseqüentemente, mantiveram a capacidade contrátil do miocárdio, diminuindo a necessidade de estímulos compensatórios, como o SNAS, na manutenção do débito cardíaco.

Em relação ao ventrículo direito, observouse um comportamento heterogêneo da porcentagem de tecido colágeno nas contagens mínima, máxima e média nos grupos experimentais G3 (tratado com 0,1mg

Ciência Rural, v.36, n.4, jul-ago, 2006. 
$\mathrm{kg}^{-1}$ ) e G4 (tratado 1,0 $\mathrm{mg} \mathrm{kg}^{-1}$ ) em relação aos G1 (normal) e G2 (coartado), pois a mesma redução parcial de tecido colágeno observada no ventrículo esquerdo repete-se na contagem mínima e média do ventrículo direito do G3 e a mesma significativa redução que ocorre no ventrículo esquerdo repete-se nas contagens máxima e média do ventrículo direito do G4. Uma hipótese para esse comportamento é que os mecanismos de ação diretos ou indiretos, na mecânica circulatória ou na liberação ou ação hormonal do carvedilol, agem mais rapidamente ou mais eficientemente no ventrículo esquerdo. Possivelmente, para agir no ventrículo direito, é necessário um tratamento com carvedilol mais prolongado.

Parece razoável afirmar que o efeito observado na redução de tecido conjuntivo só ocorre no bloqueio conjunto dos receptores $\beta 1, \beta 2$ e $\alpha 1$ adrenérgico, já que tal efeito não foi observado nos fármacos que bloqueiam os receptores $\beta$ ou $\alpha$, exclusivamente.

As baixas estimativas de correlações entre o peso do animal, o peso do coração e relação peso do coração: peso do animal permitem afirmar que as porcentagens mínima, média e máxima de tecido colágeno na parede livre dos ventrículos avaliados não foram influenciadas pelas variáveis macromorfométricas dos animais (peso do coração, peso do animal e relação peso do coração:peso do animal), diminuindo, dessa forma, o número de variáveis que poderiam interferir nos resultados do presente experimento.

\section{CONCLUSÕES}

A coartação da aorta subdiafragmática durante 20 dias aumenta a porcentagem de tecido conjuntivo nos ventrículos esquerdo e direito; o bloqueio dos receptores $\beta 1, \beta 2$ e $\alpha 1$ adrenérgico (carvedilol), em ratos com hipertrofia miocárdica concêntrica, por sobrecarga de pressão causada pela coartação da aorta subdiafragmática, reduz a porcentagem de tecido conjuntivo (colágeno) na parede livre do ventrículo direito e principalmente do ventrículo esquerdo; a redução da porcentagem de tecido conjuntivo (colágeno) na parede livre do ventrículo direito e principalmente do ventrículo esquerdo é diretamente proporcional à dose de carvedilol, ou seja, à intensidade do bloqueio dos receptores $\beta 1$, $\beta 2$ e $\alpha 1$ adrenérgico.

\section{REFERÊNCIAS}

ABRAHAMS, C. et al. Myocardial hypertrophy in Macaca fascicularis structural remodeling of the collagen matrix. Laboratory Investigation, v.56, n.6, p.676-683, 1987.

BOCCHI, F.A. et al. Effects of carvedilo (beta1, beta 2, alpha 1 bloker) on refractory congestive heart failure. Arquivos Brasileiros de Cardiologia, v.71, n.2, p.169-73, 1998.

BRAUNWALD, H. et al. Carvedilol improves function and reduces infarct size in the feline myocardium by protecting against lethal reperfusion injury. European Heart Pharmacology, v.314, n.2, p.99-107, 1996.

CHEITLIN, M.D. et al. The distribuition of fibrosis in the left ventricle in congenital aortic stenosis and coarctation of the aorta. Circulation, v.62, p.823-830, 1980.

COTRAN, R.S. et al. Patologia estrutural e funcional. 5.ed. Rio de Janeiro: Guanabara Koogan, 1996. 1268p.

GASPARI, P.G. et al. Changes in myocardial collagen in normal development and after $\beta$-blockade. Journal Molecular Cellular Cardiology, v.17, p.456-461, 1985.

HATTORI, Y. et al. Vascular effects of carvedilol, a new betaadrenoreceptor antagonist with vasodilating properties in isolated canine coronary artery. Journal of Cardiovascular Pharmacology, v.13, p.572-598, 1989.

HOFFMAN, B.B.; LEFKOWITZ, R.J. Catecolaminas, drogas simpatomiméticas e antagonistas dos receptores adrenérgicos. In: HARDMAN, J.G., LIMBIRD, J.E., Goodman \& Gilman: as bases farmacológicas da terapêutica. 9.ed. Rio de Janeiro: McGraw-Hill, 1996. Cap.10, p.146-182.

KATZUNG, B.G. Farmacologia básica e clínica. 9.ed. Rio de Janeiro: Guanabara Koogan, 2006. 1008p.

KITTLESON, M.D.; KIENLE, R.D. Smal animal cardiovascular medicine. St.Louis: Mosby, 1998. 603p.

LASTE, N.J. Cardiovascular pharmacotherapy. Veterinary Clinics of North America: Small Animal Practice, v.31, n.6, p.1231-1254, 2001.

LEFKOWITZ, et al. Neurotransmissão: os sistemas nervoso aotonômo e motor somático. In: HARDMAN, J.G.; LIMBIRD, J.E. Goodman \& Gilman: as bases farmacológicas da terapêutica. 9.ed. Rio de Janeiro: McGraw-Hill, 1996. Cap.6, p.77-102.

McTAVISH, D. et al. Carvedilol. A review of its pharmacodynamic and pharmacokinetics properties, and therapeutic efficacy in cardiovascular disease. Drugs, n.24, p.232-258, 1993.

MOORE, G.W. et al. Constituintes of the human ventricular myocardium: connective tissue hyperplasia accompanying muscular hypertrophy. American Heart Journal, v.100, n.5, p.610-617, 1980 . 
ROSSI, M.A. Fibrosis and inflamatory cells in human cronic chagasic myocarditis: scanning electron microscopy and immunohistochemical observations. International Journal of Cardiology, v.66, p.183-194, 1998.

SAS. USER'S GUIDE: Basisc and Statistic. Cary, 1995. 1686p.

SANTOS, C.M. et al. Chronic AT1 receptor blockade alter aortic nerve activity in hipertension. Hypertension, v.31, p.973-977, 1998

SAWANGKOON, S. et al. Accute cardiovascular effects and pharmacokinectis of carvedilol in healthy dogs. American Journal of Veterinary Research, v.61, n.1, p.57-60, 2000.
VAN-VLEET, J.F. et al. Pathologic alterations in hypertrophic and congestive cardiomyopathy of cats. American Journal of Veterinary Research, v.41, n.12, p.2037-2048, 1980.

VITAL, M.A.B.F. Introdução ao sistema nervoso autônomo. In: SPINOSA, H.S. et al. Farmacologia aplicada à medicina veterinária. 2.ed. Rio de Janeiro: Guanabara Koogan, 2002. p.43-58.

WATANABE, K. et al. Contribution of sympathetic nervous system activity during administration of carvedilol in rats with dilated cardiomyopathy. Jornal of cardiovascular pharmacology, v.42, p.93-97, 2003

WHY, H.J.F.; RICHARDSON, P.J. Effect of carvedilol on left ventricular function and mass in hypertension. Journal of Cardiovascular Pharmacology, v.19, n.1, p.50-54, 1992. 